\title{
TAPICES DE ESCENAS MITOLÓGICAS TEJIDOS EN LOS TALLERES BRUSELENSES DE JACQUES VAN DER BORCHT (H. 1650-H.1709-1713) Y ALBERT AUWERCX (1629-1709): NUEVA DOCUMENTACIÓN
}

\author{
Margarita García Calvo \\ Doctora en Historia del Arte \\ margagarciacalvo@gmail.com
}

\begin{abstract}
En este artículo se aporta una documentación inédita sobre dos tapicerías de Escenas mitológicas tejidas en las conocidas manufacturas de Albert Auwercx y de Jacques van der Borcht. Con la firma de este segundo tejedor se conserva en Viena (Kunsthistorisches Museum) un conjunto de tapices, que se corresponde en su mayoría con los descritos en este documento, que proporciona además interesantes novedades acerca de los pintores que participaron en el diseño de los modelos de esta serie.

Palabras clave: Jacques van der Borcht; Albert Auwercx; Charles Le Brun; Louis Van Schoor; Lucas Achtschellinck; Escenas mitológicas; Tapicerías; Bruselas; Fernando de Aragón; Juan Bautista Ysendoren.
\end{abstract}

\section{TAPESTRIES OF MYTHOLOGICAL SCENES WOVEN IN THE BRUSSELS WORKSHOPS OF JACQUES VAN DER BORCHT (C. 1650- C. 1709-1713) AND ALBERT AUWERCX (1629-1709): \\ NEW DOCUMENTATION}

This article is based on previously unpublished documentation for two tapestries of mythological scenes woven in the well-known workshops of Albert Auwercx and Jacques van der Borcht. A set of tapestries signed by van der Borcht in the Kunsthistorisches Museum, Vienna, corresponds for the most part with those described in this document, which also provides interesting information concerning the painters who participated in designing the models for this series.

Keywords: Jacques van der Borcht; Albert Auwercx; Charles Le Brun; Louis Van Schoor; Lucas Achtschellinck; Mythological scenes; Tapestries; Brussels; Fernando de Aragón; Juan Bautista Ysendoren.

En el año 1699, Fernando de Aragón y Moncada (1644-1713), $8^{\circ}$ duque de Montalto, disfruta de un buen momento económico y quiere adquirir nuevas tapicerías para su colección. Colección que había quedado muy mermada cuando después de la muerte de su padre, Luis Guillén de Moncada (1614-1672), uno de los coleccionistas más importante del siglo XVII, vende durante los años $1673-83$ casi todos los tapices que había heredado de este ${ }^{1}$.

\footnotetext{
${ }^{1}$ Delmarcel, García Calvo, Brosens, 2010: 308, doc.14
} 
Para ello, tiene a su agente en Bruselas Juan Bautista Ysendoren (¿?-1704), "primer secretario de su Magestad", que asesora a los nobles más poderosos de España en sus compras de tapices de las mejores firmas de Bruselas².

En este mismo año de 1699, Ysendoren está gestionando un encargo muy importante para el duque de Montalto: la realización de la Historia de la Casa de los Moncada, una tapicería dedicada a sus antepasados ilustres relacionados con la historia de Sicilia y sus gestas, que será tejida, en los años 1700-1703, en la manufactura de Albert Auwercx (1629-1709) y su hijo Nicolás ${ }^{3}$.

Al mismo tiempo recorre otros talleres en busca de las mejores colgaduras, de las que envía memorias puntualmente a Fernando de Aragón ${ }^{4}$. Así, visita el de Gerard Peemans (h. 1645-1725), en Bruselas, y le pasa información de una Historia de Tito y Vespasiano, diseñada por Charles Poerson (1609-1667) ${ }^{5}$ y de otra de Los Meses, tejida con modelos de David Teniers III (1638-1685) De este último tema, y tejidos en el taller de Peemans, se conservan seis tapices en el Banco de España (Madrid) ${ }^{7}$.

\section{Memoria de Jacques van der Borcht}

Dos memorias enviadas sobre tapicerías de asunto mitológico son el objeto de este artículo. Así, el 4 de agosto de 1699 recibe el duque de Montalto una memoria, firmada por "Jaques van der Beurch" "maitre tapisier", en donde se describe de una forma muy detallada una tapicería de tema mitológico, que consta de nueve piezas: "las nueve musas con el dios Palas", "el nacimiento de Adonis", "el triunfo de Bacus", "las quatro sazones del año", "el dios Jupiter y Mercurio", "la Diosa Venus con Adonis", "la Diosa Eridize con sus ninfas", "Andido (¿Endimión?) y Diana" y "El Eco que discurre con la Diosa Juno". Además de estos nueve tapices, se dice que "ay algunos dibuxos para otras piezas menores que sirven para el mismo disinio, aunque fuera para hazer 13 o catorze piezas" ". Es decir, que la serie podría ampliarse con otros temas si así lo desease el futuro comprador.

Al mismo tiempo que los temas, se mencionan también las figuras que hay en cada tapiz, las medidas y los diferentes pintores que participan en el diseño de los modelos (figs. 1 y 2).

Van der Beurch o Jacques van der Borcht (h. 1650-h. 1709-1713) perteneció a una conocida familia de tejedores de Bruselas, cuya actividad se extendió desde el último tercio del siglo XVII hasta finales del siglo XVIII ${ }^{10}$.

Una serie de ocho tapices de Representaciones mitológicas, firmada por Jacques van der Borcht se conserva en Viena (Kunsthistorisches Museum, $\mathrm{n}^{\mathrm{o}}$ inv. T LXXIX) ${ }^{11}$. Seis de las piezas

García Calvo, 2011: 284-285.

Delmarcel, García Calvo, Brosens, 2010: 284-289.

${ }^{4}$ Correspondencia entre Fernando de Aragón, duque de Montalto, y Juan Bautista Ysendoren, años 1699-1700. Archivo de Medina Sidonia (ADMS), Legajo 1106.

${ }^{5}$ Reyniés, 1997: 171-184. Brosens, 2008: 174-177; 2012: 10, apéndice 3. Delmarcel, 2010:114-123. García Calvo, 2014: 80-83, 88-90.

${ }^{6}$ Vlieghe, 1950-60: 78-102. Bauer, 1977: 65-84. Blazkova, 1981:203-220. Forti Grazzini, 1998: 136-146. Hartkamp-Jonxis, Smit, 2004: 131-141; Schmitz-Von Ledebur, 2008a: 246-252. García Calvo, 2014: 84-87.

${ }^{7}$ Ramírez Ruiz, 2013: 376, 507-511. García Calvo, 2014: 84-87.

${ }^{8}$ ADMS, Legajo 1106, carta del 5 de agosto de 1699, enviada desde Bruselas por Isendoren al duque de Montalto. Con ella se adjuntan las dos memorias de tapicería objeto de este artículo. Apéndice.

9 ADMS, Legajo 1106. Apéndice.

${ }^{10}$ Delmarcel, 1999: 363. Brosens, 2004: 344.

${ }^{11}$ Bauer, 1991: 81-86. Delmarcel, 1999: 307. Brosens, 2004: 93, 518-519; 2008: 445. Schmitz-Von Ledebur, Katja, "Tapisserien mit mythologischen Darstellungen", en Vom Mythos der Antike. Catálogo a cargo de Wilfried Seipel. Milán/Viena, 2008, pp. 62-65, nºs. 17-18. Schmitz-Von Ledebur, 2008-2009: 220-223. 


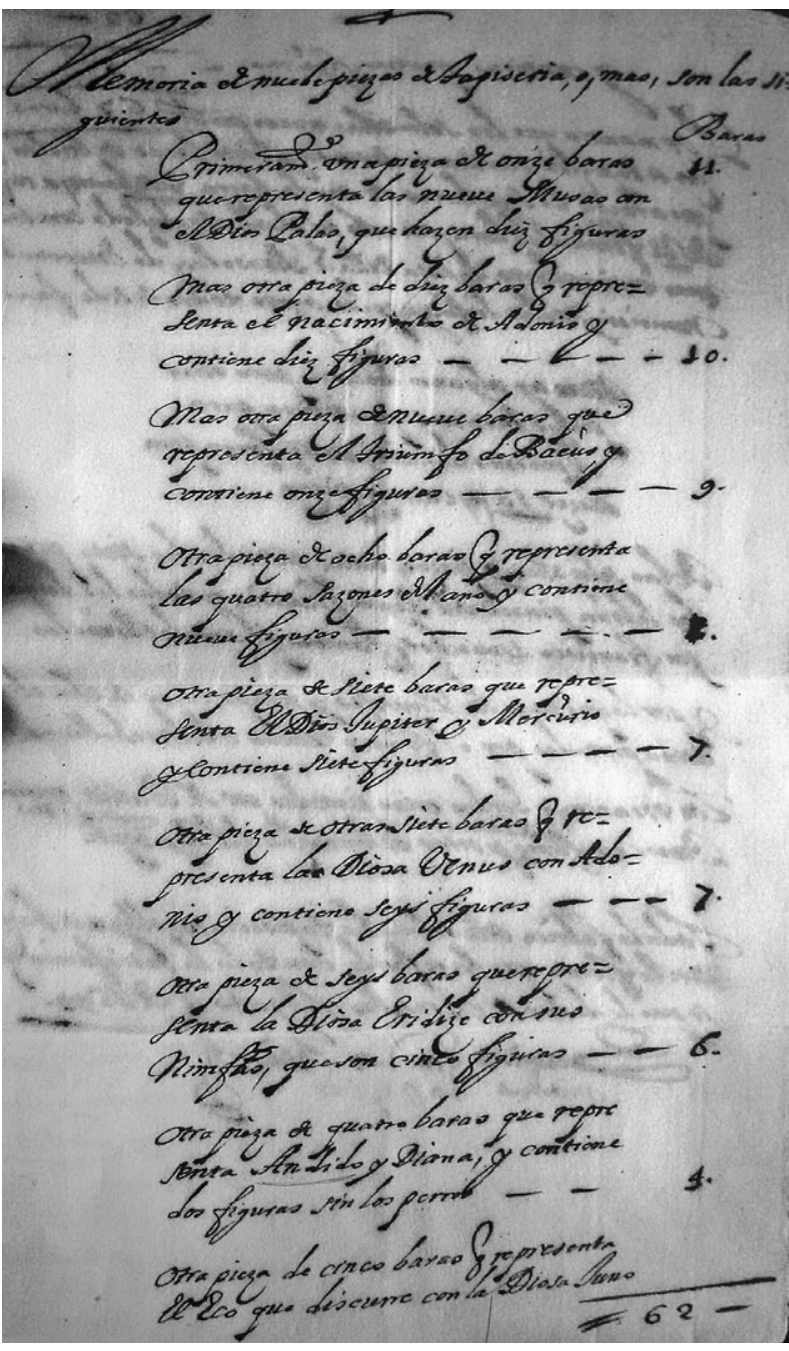

Fig. 1. Memoria de "Jaques van der Beurch", 4 de agosto de 1699. Archivo de Medina Sidonia. Legajo 1106. que se describen en esta memoria de 1699 se pueden poner en relación con otras seis de este conjunto de Viena en cuanto a la identificación de los temas: Minerva siendo recibida por las musas ( ${ }^{\circ} 1$ de la memoria), Triunfo de Baco y Ariadna ( $\left.\mathrm{n}^{\circ} 3\right)$, Las cuatro estaciones del año haciendo sacrificios a Apolo $\left(\mathrm{n}^{\circ}\right.$ 4), Venus y Adonis ( ${ }^{\circ}$ 6), La muerte de Euridice $\left(\mathrm{n}^{\mathrm{o}} 7\right)$ y Diana y Endimión $\left(\mathrm{n}^{\mathrm{o}}\right.$ 8) (figs. 3, 4 y 5). Las piezas citadas en la memoria con los $\mathrm{n}^{\circ} 2$, "nacimiento de Adonis", no 5, "Jupiter y Mercurio" y la $\mathrm{n}^{\mathrm{o}}$ 9, "el Eco que discurre con la diosa Juno", no parecen corresponderse con ninguna de Viena. Por otro lado, dos de los tapices de la serie de Viena, Flora coronada por Céfiro y Cupido y Mercurio entregando a Baco niño a las ninfas en el monte Nysa, no se mencionan en la memoria.

La memoria de "Jaques van der Beurch" proporciona una información sobre esta tapicería de asunto mitológico que no es frecuente en documentos de este tipo, ya que da los nombres de los diferentes artistas que participan en los diseños. Así, dice que "delas sobredhas 9 piezas, estan pintadas las siete por Carlos Lebrûn pintor del Rey de Francia, y todas las demas por francisco vanschor, famoso pintor de Bruselas"12.

El pintor francés Charles Le Brun (1619-1690) fue autor de los modelos de conocidas tapicerías, como la de la Historia de Meleagro y Atalanta, la de Los Cuatro Elementos o la Historia del Rey, serie esta última en honor de Luis XIV (1638-1715) y de su corte, de la Manufactura Real de los Gobelinos, pero no se le había considerado el pintor de las Escenas mitológicas de Viena ${ }^{13}$, aunque se ha señalado en varias ocasiones la influencia francesa de los diseños de esta serie. En concreto, se ha mencionado un grabado de Nicolas Poussin (1594-1665) El nacimiento de Baco (Cambridge, Mass., Fogg Art Mu-

12 ADMS, Legajo 1106. Apéndice.

${ }^{13}$ Esta atribución que hace la memoria firmada por Van der Beurch a Le Brun como autor de los diseños de siete tapices quizás haya que tomarla con prudencia, teniendo en cuenta que, en ocasiones, los agentes recomendaban a sus clientes obras que tuviesen una reputación y, en este momento, Le Brun era un pintor muy conocido en Europa mientras que la fama de Van Schoor, tributario de la pintura francesa, era solo local. 


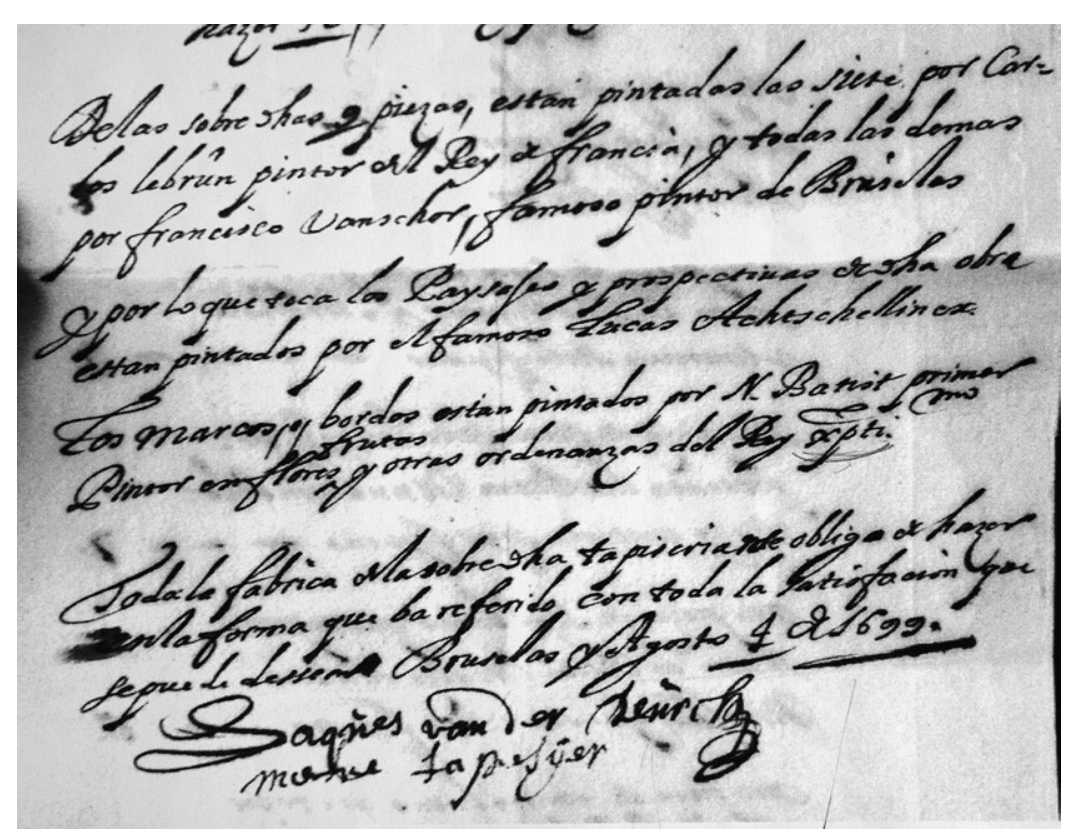

Fig. 2. Memoria de "Jaques van der Beurch", 4 de agosto de 1699. Archivo de Medina Sidonia. Legajo 1106.

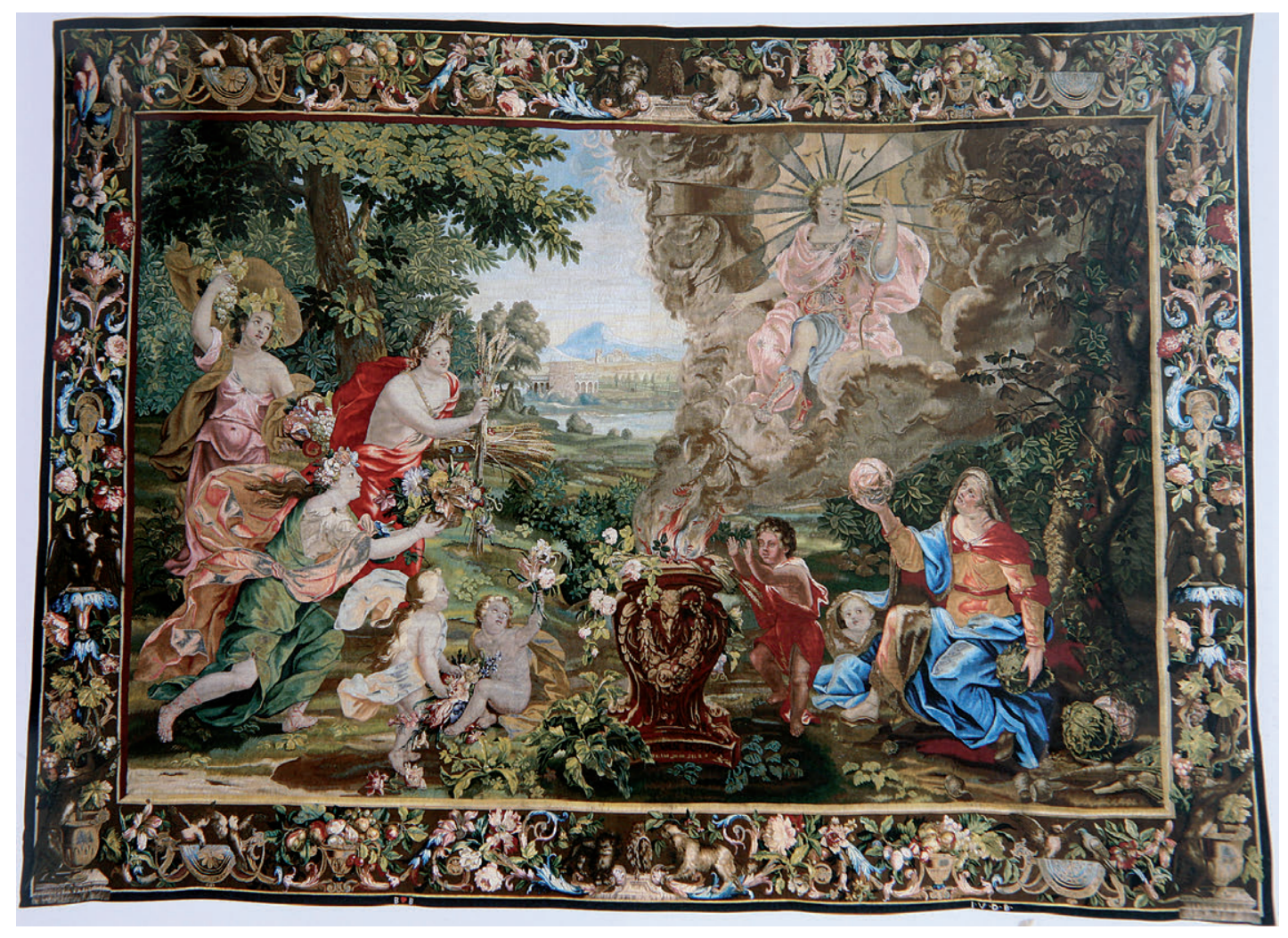

Fig. 3. Tapiz de "Las cuatro estaciones hacen ofrendas a Apolo", de la serie Representaciones mitológicas. Tejido en el taller de Jacob van der Borcht, Bruselas, h. 1695. 352 x 490 cm. Kunsthistorisches Museum, Viena. 


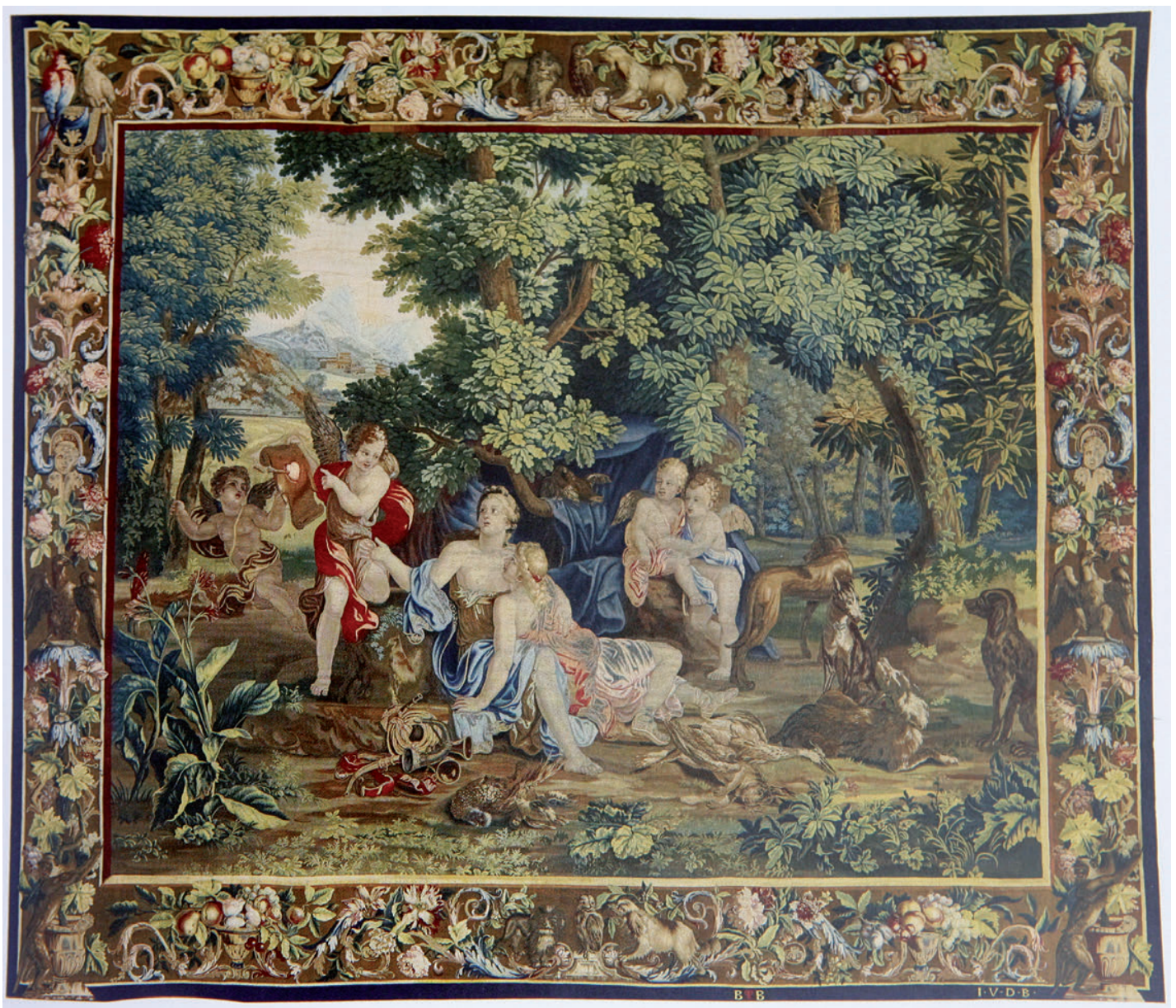

Fig. 4. Tapiz de "Venus y Adonis", de la serie Representaciones mitológicas. Tejido en el taller de Jacob van der Borcht, Bruselas, h. 1695. 350 x 410 cm. Kunsthistorisches Museum, Viena.

seum $)^{14}$ como inspiración del tapiz de Viena Mercurio entregando a Baco niño a las ninfas ${ }^{15}$, o al mismo Le Brun por la similitud que presentan las figuras del Triunfo de Baco y Ariadna de Viena con las de La Terre (París, Mobilier National), tapiz de los Elementos, serie diseñada por Le Brun y tejida en la Manufactura Real de los Gobelinos de París en el año $1666^{16}$.

Dice la memoria también que de los nueve tapices, dos estarían pintados por "francisco vanschor". El nombre de "francisco" parece ser un error y se podría estar refiriendo a Louis Van Schoor (h. 1650-1702) ${ }^{17}$.

${ }^{14}$ Thuillier, 1975: lám. LIV. Para la relación de Poussin y la tapicería ver a Bayard, Brejon De Lavergnée, Chassey, 2011.

${ }^{15}$ László, 1981: 90, figs. 94-95. Bauer, 1991: 81-82. Schmitz-Von Ledebur, 2008-2009: 220-221.

${ }^{16}$ Knothe, 2008: 356-364. Vittet, Brejon De Lavergnée, 2010: 129-133.

${ }^{17}$ Agradezco esta observación a Guy Delmarcel. 


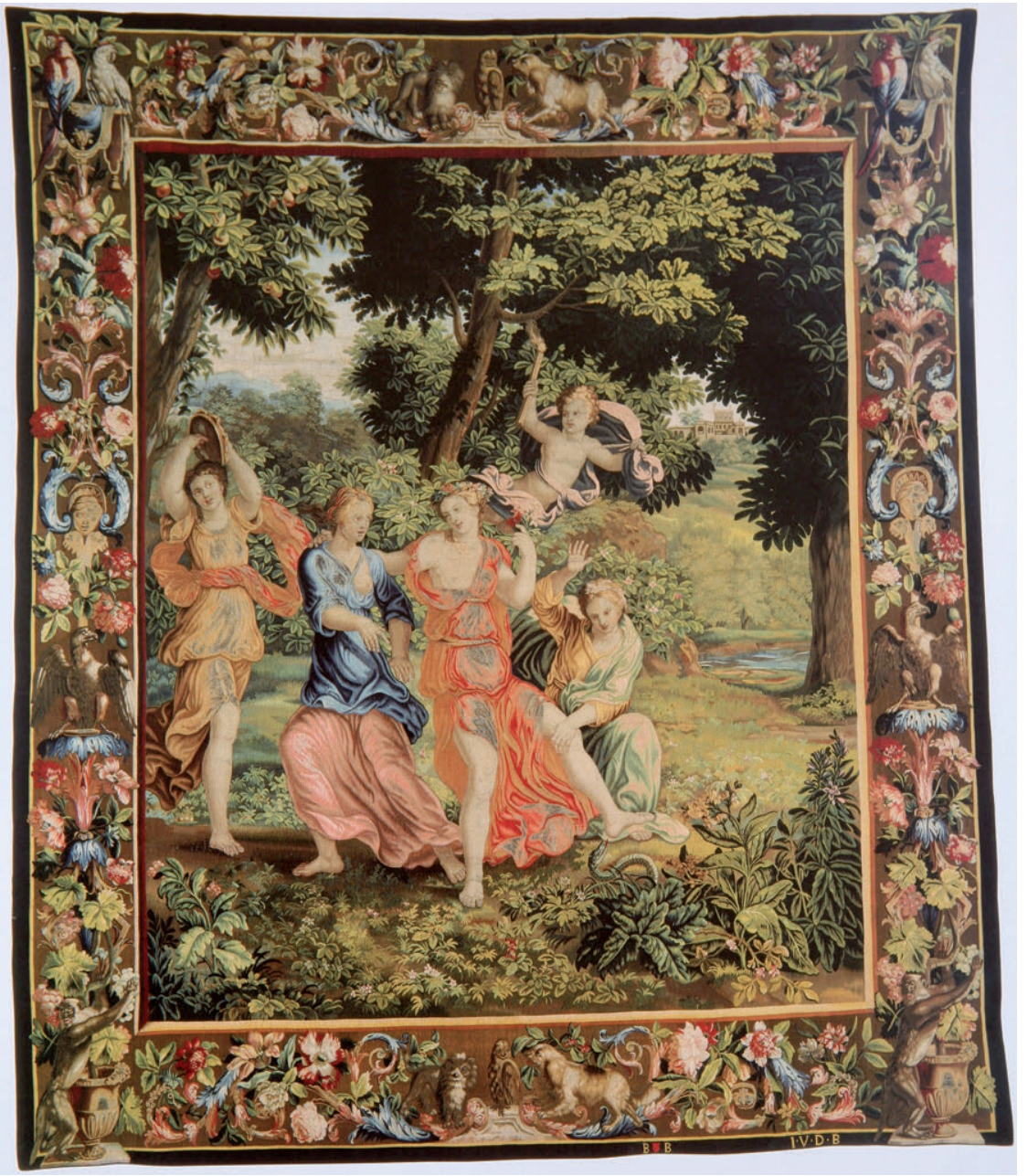

Fig. 5. Tapiz de "Eurídice mordida por la serpiente", de la serie Representaciones mitológicas. Tejido en el taller de Jacob van der Borcht, Bruselas, h. 1695. $350 \times 300 \mathrm{~cm}$. Kunsthistorisches Museum, Viena.

Louis Van Schoor fue un pintor del barroco tardío, con aires ya clásicos, formado en la escuela del pintor David Teniers III , que diseñó al menos quince conjuntos de tapices a lo largo de su carrera artística, entre ellos el muy conocido de Perseo y Andrómeda (Kunsthistorisches Museum, Viena), y que muy posiblemente participó en el diseño de la célebre serie de 29 paños, dedicada a la Vida de Cristo, el Triunfo de la Eucaristía y los Doce Apóstoles (Catedral de Valetta) ${ }^{18}$.

Dos tapices de las Escenas mitológicas de Viena vienen firmados, en efecto, por Van Schoor: Las cuatro estaciones del año haciendo sacrificios a Apolo y Minerva siendo recibida por las musas.

La mención que hace la memoria, por otro lado, del "famoso Lucas Acheschellinex" como pintor de "los paysajes y prospectivas de dicha obra"19, no hace sino confirmar documentalmente lo que venía siendo una atribución para la serie de Viena.

Lucas Achtschellinck (1626-1699), al que se le garantizaron privilegios como pintor de paisajes en 1689, participó en los diseños de varias tapicerías pintando los paisajes. A veces en

\footnotetext{
${ }^{18}$ Delmarcel, 1999: 308-309.

19 ADMS, Legajo 1106. Apéndice.
} 
colaboración con Van Schoor, como en la Historia de Perseo y Andrómeda, citada anteriormente, o en estas Escenas mitológicas de Viena ${ }^{20}$.

Por último, esta memoria de "Van der Beurch" atribuye la autoría de los "marcos o bordes" a "N. Batist primer pintor en flores, frutos y otras ordenanzas del Rey xptimo" ${ }^{21}$. Esta última palabra es la abreviatura de "cristianísimo", denominación que se aplicaba a los reyes franceses, refiriéndose en este caso al rey Luis XIV.

Del pintor N. Batist no tenemos ninguna referencia, nos es totalmente desconocido. ¿Podría haber aquí un error en el nombre como hemos visto, por ejemplo, con "francisco van Schoor?

Son algunos los pintores cuyo nombre coincide en parte con el de la memoria, sin que esto suponga en absoluto un intento de atribución. Uno de ellos fue Jean-Baptiste Monnoyer (1636-1699), Baptiste para sus contemporáneos, que fue muy conocido por sus pinturas de floreros y bodegones, tan apreciados por el rey Luis XIV que sesenta de sus lienzos terminaron formando parte de la colección real. A partir de la década de 1660 colaboró en los grandes programas decorativos de Le Brun para las residencias reales. Además, trabajó desde 1666 para la manufactura de los Gobelinos pintando secciones detalladas de cartones según las necesidades de los liceros ${ }^{22}$. También es conocido su trabajo para la manufactura de Beauvais, colaborando en los modelos de series de gran éxito como los Grutescos y la Historia del emperador de China, que se tejieron repetidamente durante más de cuatro décadas, desde 1688 hasta alrededor de 1732, cuando el deterioro de los cartones los hizo inservibles ${ }^{23}$.

Es interesante tener presente también que las cenefas del conjunto de Viena recuerdan a las que se utilizaron en Francia, en tapices tejidos en Beauvais especialmente.

Otro pintor de la época con alguna coincidencia en cuanto al nombre fue Jean-Baptiste Belin (1653-1715), que sucedió a Monnoyer como pintor de flores en la manufactura de los Gobelinos. También obtuvo el favor de la corte, en particular del rey Luis XIV, lo que se tradujo en encargos para los palacios de Versalles o Fontainebleau.

Por los mismos diseños de la serie de Viena se tejieron otras ediciones, como lo demuestran los tapices que hay en diferentes colecciones. Por ejemplo, Las cuatro estaciones hacen ofrendas a Apolo y el Triunfo de Baco y Ariadna (fig. 6), que forman parte de la colección del Castillo Wawel de Cracovia y que se consideran tejidos en Bruselas hacia 1700, posiblemente en el taller de Jacques van der Borcht. Carecen de cenefas y presentan ligeras variantes, en cuanto a los diseños, de los de Viena, como son las diferencias en el paisaje de ambos y también en el segundo tapiz se han añadido figuras en el lado derecho, al mismo tiempo que faltan en el izquierdo las del Sileno montado en un asno y sus acompañantes, suprimidos probablemente al mismo tiempo que las cenefas ${ }^{24}$.

También se conserva en el Museo de Poznan una pieza del Triunfo de Baco y Ariadna que sigue el modelo ya mencionado. Tejida hacia 1700 en la misma manufactura de Van der Borcht tiene una cenefa muy similar a la del tapiz de Viena ${ }^{25}$ (fig. 7). El mismo paño del Triunfo forma parte de la colección de tapices del Bayerisches Nationalmuseum (Munich) ${ }^{26}$. Con la misma bordura de Viena fue subastado otro Triunfo de Baco y Ariadna por Sotheby's (New York, 5 de junio de 1997) ${ }^{27}$ (fig. 8). En estos tapices se puede apreciar la similitud de las figuras con las de La Terre

\footnotetext{
${ }^{20}$ Delmarcel, 1999: 308-309. Brosens, 2004: 97-98.

${ }^{21}$ ADMS, Legajo 1106. Apéndice.

${ }^{22}$ Bremer-David, 2008: 428.

${ }^{23}$ Coural, Gastinel-Coural, 1992: 21-28, figs. 7, 8, 9, 10 y 11. Bremer-David, 2008: 427-439.

${ }^{24}$ Hennel-Bernasikowa, 2000: 171-174. Piwocka, 2011: 250-251.

${ }_{25}$ Wasilkowska, 1971: 94-97, figs. 46-47.

Hennel-Bernasikowa, 2000: 316-317.

${ }^{26}$ Schneider, 1924: 70-72, fig. 13

27 Ver el catálogo de Sotheby's European Works of Art, Arms and Armour, Furniture and Tapestries, New York, 1997 : 108-109, fig. 287.
} 


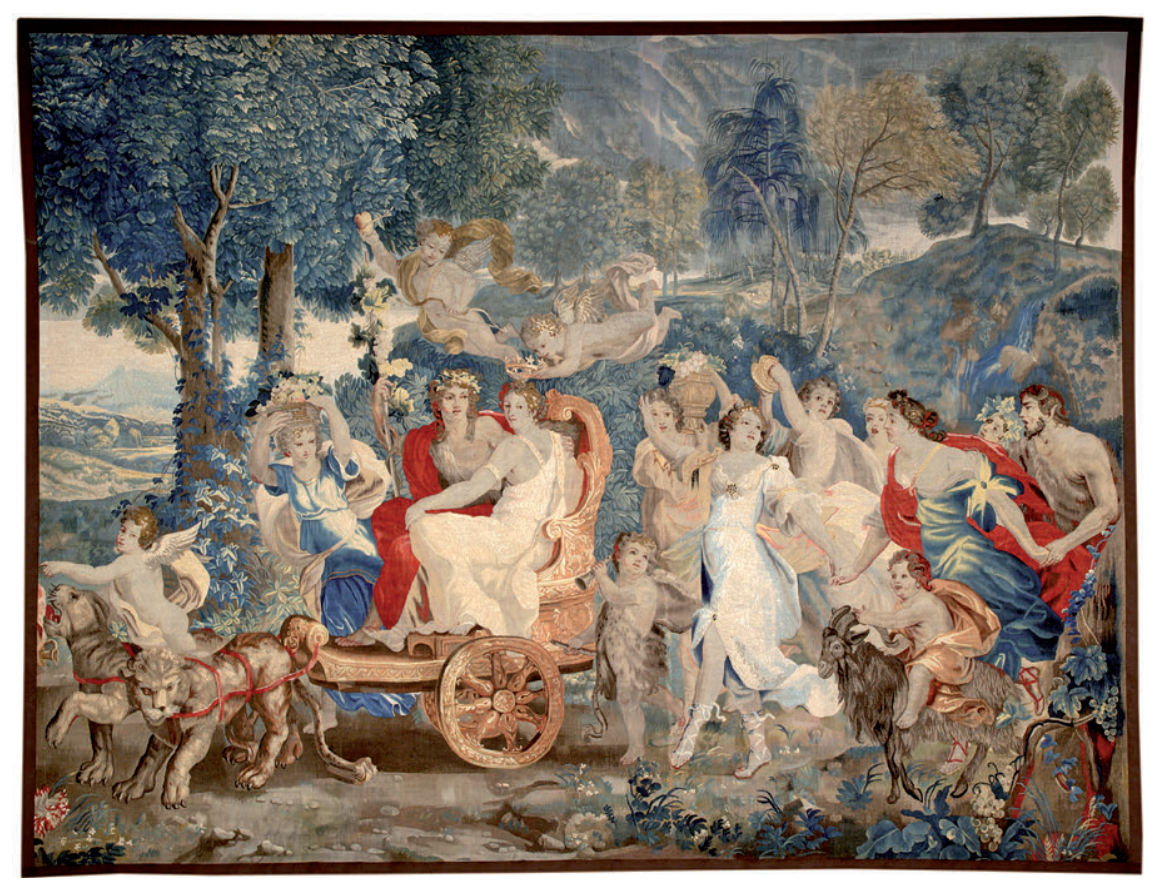

Fig. 6. Tapiz del "Triunfo de Baco y Ariadna". Atribuido al taller de Jacob van der Borcht, Bruselas, h. 1700. 270 x 346 cm. Zamek Królewski na Wawelu, Cracovia.

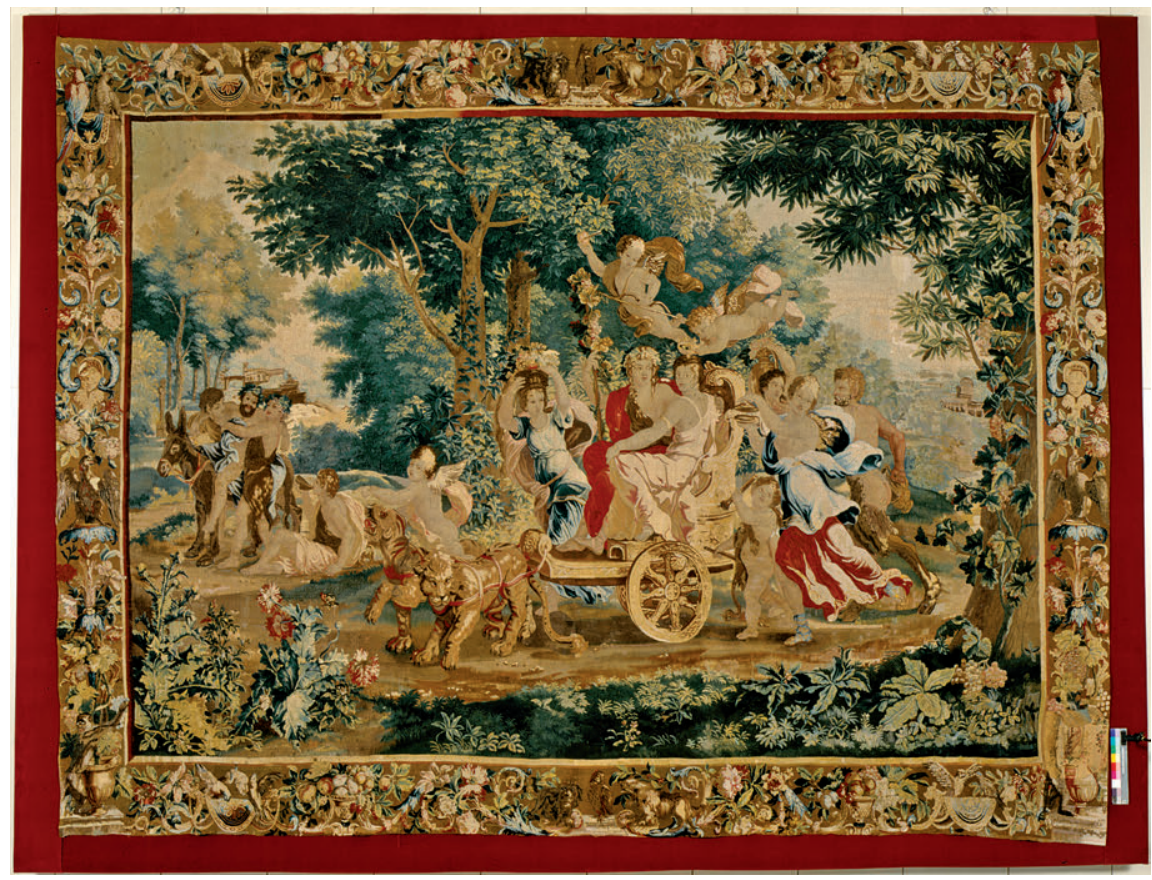

Fig. 7. Tapiz del "Triunfo de Baco y Ariadna". Tejido en el taller de Jacob van der Borcht, Bruselas, h. 1700. 370 x $585 \mathrm{~cm}$. Muzeum Narodowew Poznaniu, Poznan. 


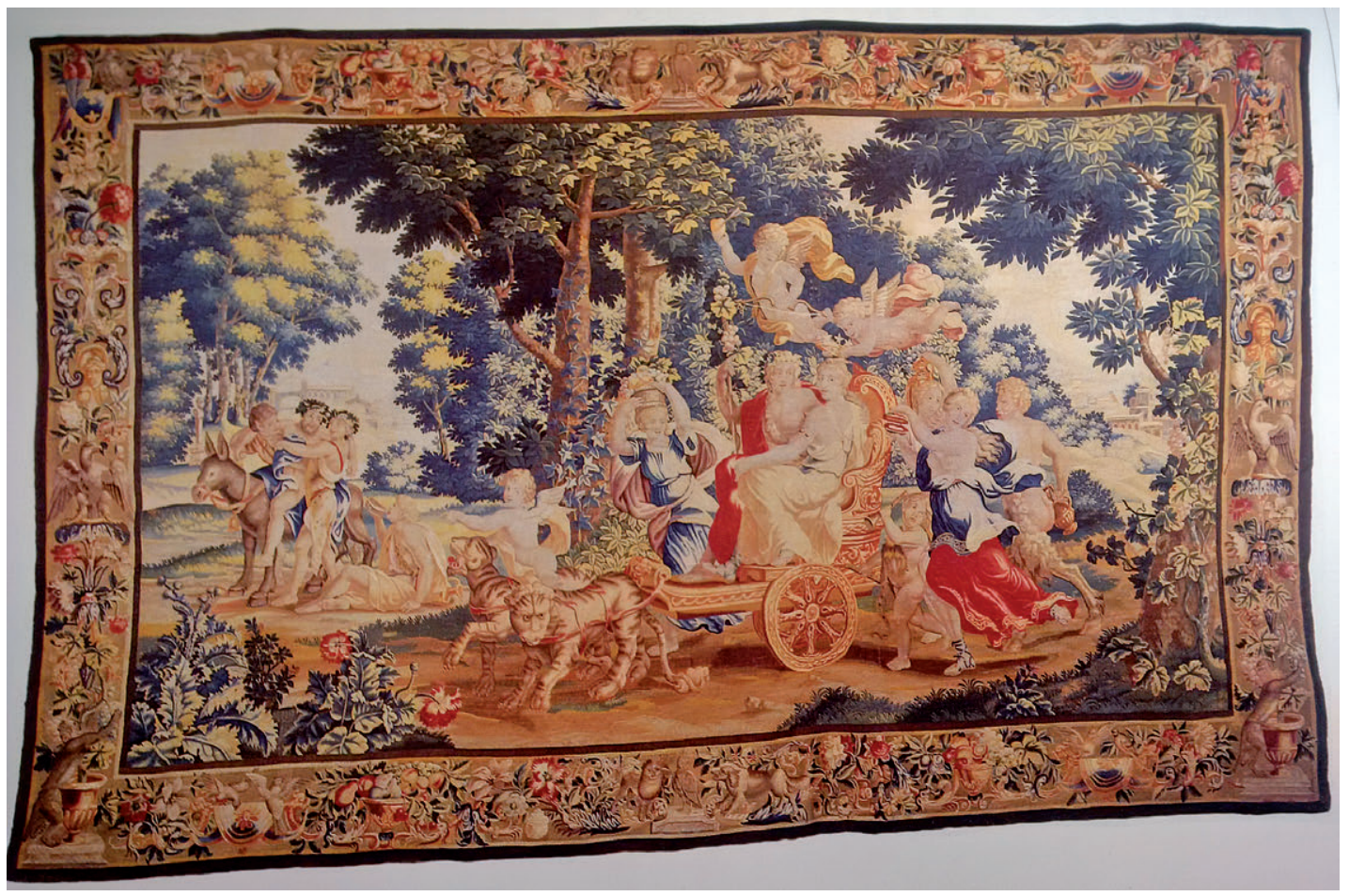

Fig. 8. Tapiz del "Triunfo de Baco y Ariadna". Atribuido al taller de Jacob van der Borcht, Bruselas, h. 1700 Vendido en Sotheby's, Nueva York, en 1997.

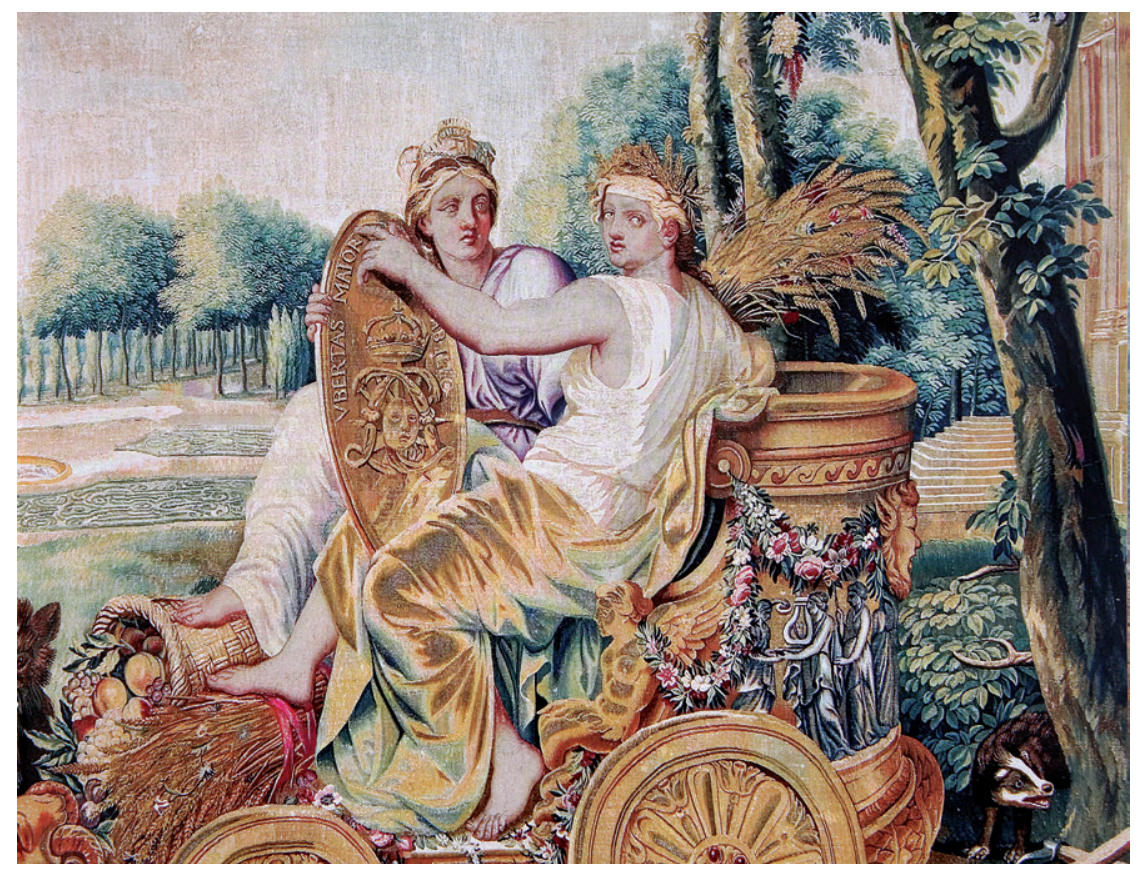

Fig. 9. Detalle del tapiz "La Terre", de la serie de Los elementos. Tejido en los Gobelinos, en el taller de De La Croix, h. 1667. Diseño de Charles Le Brun. 350 x 630 $\mathrm{cm}$. Mobilier National, París. 
de Lebrun, como ya se ha dicho (fig. 9). En el año 2000 vuelve a salir al mercado otro tapiz del mismo asunto en la misma casa de subastas, pero con una cenefa diferente al vienés ${ }^{28}$.

La misma pieza del Triunfo de Baco y Ariadna, pero con una bordura distinta que incorpora las armas de la familia Colón en las esquinas, fue propiedad de Pedro Núñez de Colón, duque de Veragua. Posteriormente pasaría a formar parte de la colección Berwick-Alba ${ }^{29}$ y en el año 1997 fue subastada por Christie (Nueva York, 29 de enero, lote 138) (fig. 10).

De los otros temas de esta serie mitológica también se conservan ejemplares, como es el caso de Mercurio entregando a Baco niño a las ninfas (Museo de Artes Decorativas de Budapest), tejido por Van der Borcht y con la misma cenefa que la tapicería vienesa ${ }^{30}$.

Inspirándose en estos modelos de las Escenas mitológicas, tejidas en la manufactura de Van der Borcht de Bruselas, aún se teje en Audenarde, hacia 1715, una serie ${ }^{31}$.

\section{Memoria de Albert Auwercx}

En otra de las memorias que recibe Fernando de Aragón, en 1699, se menciona otra tapicería "des metamorphoses d'Ovide", que "tiene entre las manos" Albert Auwercx ${ }^{32}$.

Auwercx estuvo al frente de una de las manufacturas más importantes de Bruselas, en donde se tejieron conjuntos tan conocidos como la Historia de la Casa de los Moncada, citada al principio de este trabajo. Fue un reconocido especialista también en tejer tapices heráldicos para la nobleza española, de los que conservan ejemplares, entre otros, la familia Medinaceli en el Hospital de Tavera (Toledo). También vemos la firma de Auwercx, entre otros ejemplos, en una Historia de Moisés que se conserva en la catedral de Toledo etc. ${ }^{33}$

Este conjunto "des metamorphoses d'Ovide" en el que trabaja Auwercx, y del que se proporcionan en la memoria los temas, las medidas y el precio, está compuesto de ocho piezas, aunque de la número siete no se dice el asunto, y son las siguientes: "Eurídice mordida por la serpiente en un pie" ( $\left.\mathrm{n}^{\circ} 1\right)$, "el rapto de Adonis" $\left(\mathrm{n}^{\circ} 2\right)$, "la danza de las ninfas báquicas en honor de Baco" $\left(\mathrm{n}^{\circ}\right.$ 3), "Acis y Galatea" ( $\left.\mathrm{n}^{\circ} 4\right)$, "Mercurio retenido por Aglauro" $\left(\mathrm{n}^{\circ} 5\right)$, "Ceres buscando a Proserpina" (n $\mathrm{n}^{\mathrm{o}}$ ), "Pomona y Vertuno" $\left(\mathrm{n}^{\mathrm{o}} 8\right)^{34}$. El tapiz que haría el número 7 y que falta en la memoria podría ser el de Palas y Aracné, si nos atenemos a la descripción, hecha en 1700, de una serie bruselesa con los mismos temas y coincidente en la altura, seis anas ${ }^{35}$.

En el memorial del marchante de tapicería de Amberes, Nicolaas Naulaerts, se menciona otra tapicería de estas verduras con personajes de las Metamorfosis de Ovidio, también compuesta de ocho tapices cuyos episodios coinciden con los citados en el documento de Auwercx, además del de Palas y Aracné que no aparece en este, como ya se ha dicho ${ }^{36}$.

${ }^{28}$ La fecha de la subasta fue el 19 de mayo de 2006, lote 42.

${ }^{29}$ Ramírez Ruiz, 2013: 548.

${ }^{30}$ László, 1981: 90, fig. 94.

${ }^{31}$ De Meûter, 1999: 222-228. Recientemente también ha sido subastado en Sothebys (Londres, 6 de noviembre de 2014) un tapiz del "Triunfo de Baco y Ariadna", tejido en Audenarde en el siglo XVIII.

32 ADMS, Legajo 1106. Apéndice.

33 Delmarcel, 1999: 363. Delmarcel, García Calvo, Brosens, 2010: 283-294. Brosens, 2012: 184-188.

34 ADMS, Legajo 1106. Apéndice.

${ }^{35}$ Esta serie bruselesa incluye los siguientes temas: “Adonis devient la proie de Venus, Eurydice piquée par le serpent, les Bachanales, la Rencontre d'acis et Galatée, Mercure retenu par Aglauros, Cérés cherchant sa fille Proserpine, Pallas et Arachné, Pomone et Vertumne" (El rapto de Adonis, Eurídice mordida por la serpiente, las Bacanales, El encuentro de Acis y Galatea, Mercurio retenido por Aglauro, Ceres buscando a Proserpina, Palas y Aracne y Vertuno y Pomona). Delmarcel, 1999: 305.

${ }_{36}$ " Le rapt d'Adonis par Venus, Euridice est mordu d'une couleuvre, Le Bacchinal, Acis le berger vient auprés de Galathée, Aglaure repouse Mercure, Ceres chercha sa fille Proserpine, Pallas et Ariadne, et Vertumne et Pomona". De Meûter, 2003: 138. 


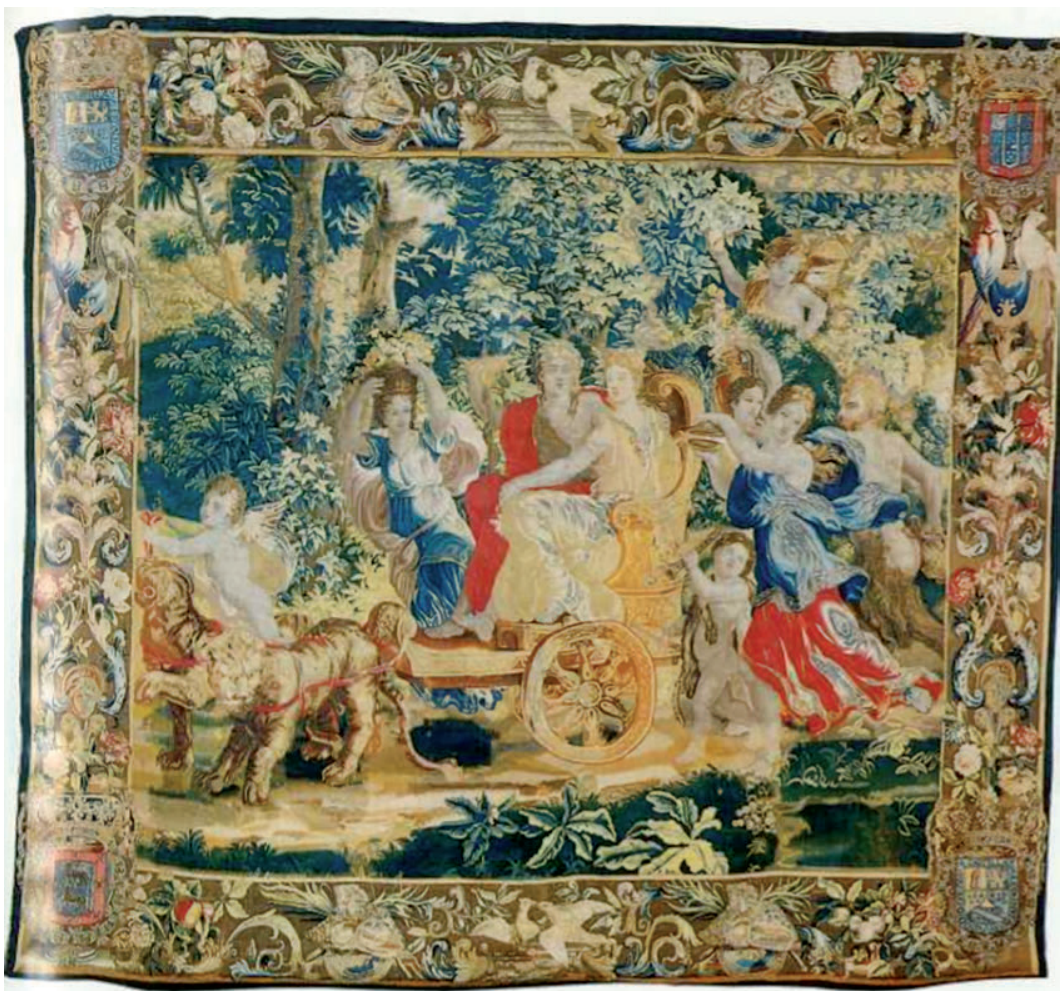

Fig. 10. Tapiz del "Triunfo de Baco y Ariadna". Tejido en Bruselas, h. 1700. $299 \times 325 \mathrm{~cm}$. Vendido en Christie, Nueva York, en 1997.

Estas series mitológicas fueron muy populares en los talleres de Bruselas y de Amberes a finales del siglo XVII y principios del XVIII, siendo el precio de estos tapices diferente según que fuesen tejidos en una ciudad u otra. Mientras que en Amberes el ana costaba entre 12 y 14 florines, en Bruselas estaba entre 18 y 20, cantidad que estaba relacionado con la finura del tejido generalmente ${ }^{37}$. El precio de la tapicería en la memoria de Auwercx es de 17 florines el ana, cantidad algo baja para Bruselas.

Un ejemplo de tapiz de este tipo podría ser el de Euridice mordida por una serpiente (Coll. Royal Manufacturers De Wit), tejido hacia 1700 en Amberes o Bruselas según un diseño atribuido a Peter Ykens (1648-1695) y a Pieter Spierinckx ${ }^{38}$ (1635-1711) (fig. 11).

Después de haberse interesado por estas tapicerías, el duque de Montalto decide no adquirir ninguna y centrarse en la gran tapicería de la Historia de la Casa de los Moncada, que será tejida por el mismo Albert Auwercx, como ya se ha dicho: " no habiendo en las memorias que vm me remite ninguna tapizeria acabada ni de la forma que yo lo deseo, aunque hay algunos paños que me han parecido buenos, habiendo de esperar a que se acaben no entro en ninguna, por que mas quiero se trabaje en la dela Historia de mi Casa y esperarla, que no aguardar por ninguna de estas obras, que la comprara si estubiera hecha, y me contentara, y en esta inteligencia si vm la descubriese me lo avisase..." $"$.

${ }^{37}$ De Meûter, 2003:139

${ }^{38}$ Van Tichelen, Delmarcel, 1989: 54-55. Delmarcel, Volckaert, 1995: 60-61. Delmarcel, 1999: 306. Brosens, 2008:205.

${ }^{39}$ ADMS, Legajo 1106. Carta con fecha 7 de septiembre de 1699, escrita por Fernando de Aragón a Juan Bautista Ysendoren. Ver a García Calvo, 2014: nota 34. 


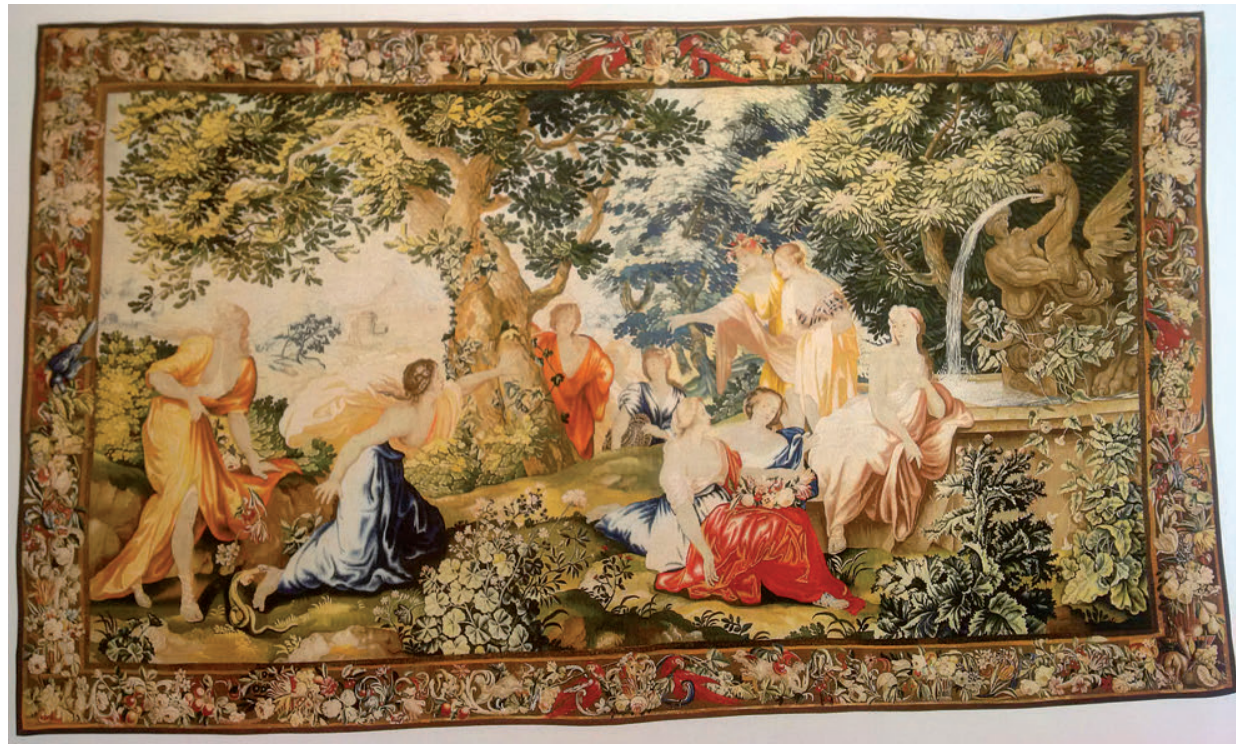

Fig. 11. Tapiz de "Eurídice mordida por una serpiente". Tejido en Bruselas o Amberes, h. 1700. 325 x 465 cm. Coll. Royal Manufacturers De Wit, Bélgica.

\section{APÉNDICE}

"Memoria de nuebe piezas de tapicería, o mas, son las siguientes"

Baras

Primeramente una pieza de onze baras que representa las nueve musas con el Dios Palas, que hazen diez figuras

Mas otra pieza de diez baras que representa el nacimiento de Adonis y contiene diez figuras Mas otra pieza de nueve baras que representa el triunfo de Bacus y contiene onze figuras Otra pieza de ocho baras que representa las quatro sazones del año y contiene nuebe figuras Otra pieza de siete baras que representa el Dios Jupiter y Mercurio y contiene siete figuras Otra pieza de otras siete baras que representa la Diosa Venus con Adonis y contiene seys figuras Otra pieza de seys baras que representa la Diosa Eridize con sus Nimfas, que son cinco figuras Otra pieza de quatro baras que representa Andido y Diana, y contiene dos figuras sin los perros Otra pieza de cinco baras que representa El Eco que discurre con la Diosa Juno y contiene quatro figuras

Por manera que las sobredichas nueve piezas de tapizeria contienen sesenta y siete baras de buelta, que arazon de seys baras de cayda hazen 402 baras, a razon de 21 florines la bara, sin oro ni plata, trabajada con los mas vivos colores de esta villa de Bruselas, de encarnado cramoise y color de fuego, y los ayres todos de seda fina.

Mas ay algunos dibuxos para otras piezas menores que sirven para el mismo disinio, aunque fuera para hazer 13 , o, catorze piezas.

Delas sobre dichas 9 piezas, estan pintadas las siete por Carlos Lebrun pintor del Rey de Francia, y todas las demas por Francisco Vanschor, famoso pintor de Bruselas

Y por lo que toca los Paysajes y prospectivas de dicha obra estan pintados por el famoso Lucas Acheschellinex.

Los marcos, o, bordes estan pintados por N. Batist primer pintor en flores, frutas y otras ordenanzas del Rey xptimo.

Toda la fabrica dela sobredicha tapicería se obliga a hazer en la forma que ha referido con toda la satisfacion 
que se puede dessear. Bruselas. Agosto 4 de 1699.

Jaques van der Beurch

maitre tapissier

(ADMS, leg. 1106. 4 de agosto de 1699)

"Memoire d'une tapisserie que le susdit. tappissier Auwercx at entre les mains; hors des metamorphoses d'Ovide de six aunes d'hauteur consistant en huit pieces ".

1'honneur de Bacus

$\mathrm{N}^{\mathrm{o}}$ 4- Acis et Galathee $\quad 6 \quad 1 / 4$

$\mathrm{N}^{\mathrm{o}}$ 5- Mercurius retenu de Glauca_— $51 / 4$

$\mathrm{N}^{\mathrm{o}}$ 6- Ceres cherchant Proserpine —u $43 / 4$

$\mathrm{N}^{\mathrm{o}} 7-$

No 8 - Pomona y Vertumne_- $31 / 4$

Ensemble en tour $\quad 46 \quad 7 / 8$

A 17 florins l'aune 4761

5 multiplie par 6

(ADMS, leg. 1106. 5 de agosto de 1699)

Memoria de una tapicería que el susodicho tapicero Auwercx tiene entre las manos; inspirada en las metamorfosis de Ovidio de seis anas de altura consistente en ocho piezas.

$\mathrm{N}^{\mathrm{O}}$ 1- Eurídice mordida por la serpiente en un pie — $\quad 3 / 8$

$\mathrm{N}^{\circ}$ 2- El rapto o levantamiento de Adonis_ $81 / 2$

$\mathrm{N}^{0}$ 3- La danza de las ninfas Báquicas en honor de Baco _

$\mathrm{N}^{\circ}$ 4- Acis y Galatea $\quad 6 \quad 1 / 4$

$\mathrm{N}^{0}$ 5- Mercurio retenido por Aglauro — $51 / 4$

$\mathrm{N}^{\circ}$ 6- Ceres buscando a Proserpina_— $43 / 4$

$\mathrm{N}^{\mathrm{o}} 7-$

No 8 - Pomona y Vertuno — $31 / 4$

A 17 florines el ana 4761

En conjunto alrededor de $46 \quad 7 / 8$

5 multiplicado por 6

$281 \quad 1 / 4$

(ADMS, leg. 1106. 5 de agosto de 1699)

\section{BIBLIOGRAFÍA}

Bayard, Marc / Brejon de Lavergnée, Arnauld / Chassey, Éric (2011): Poussin et Moïse, du dessin á la tapisserie, Roma. Bauer, Rotrand (1977): Tapisseries bruxelloises au siécle de Rubens du Kunsthistorisches Museum, Vienne et des Museés royaux d'Art et d'Histoire, Bruselas.

Bauer, Rotrand (1991): “Tapisserien”. Wohnen im Schloss: Tapisserien, Möbel, Porzellan und Kleid er aus drei Jahrhunderten, cat. exp., Scholss Halbturn, pp. 81-86.

Blazkova, Jarmila (1981): "Deux tentures des Mois á Prague”. En: Artes Textiles, 10, pp. 203-220.

Bremer-David, Charissa (2008): "La Real Manufactura de Tapices de Beauvais, 1664-1715". En: Campbell, Thomas P. (responsable científico): Hilos de esplendor. Tapices del Barroco, cat. exp., Madrid, Patrimonio Nacional, pp. 427-439.

Brosens, Koenraad (2004): A contextual study of Brussels Tapestry, 1670-1770. The dye works and Tapestry workshop of Urbanus Leyniers (1674-1747), Bruselas. 
Brosens, Koenraad (2008): European Tapestries in the Art Institute of Chicago, Londres.

Brosens, Koenraad (2012): "Revisiting Brussels tapestry, 1700-1740: New data on Tapissiers Albert Auwercx and Judocus de Vos«. En: Textile History, 43, pp. 183-199.

Catálogo (1997): European works of Art, Arms and Armour, Furniture and Tapestries, Sotheby's, Nueva York.

Coural, Jean / Gastinel-Coural, Chantal (1992): Beauvais. Manufacture nationale de Tapisserie, París.

Delmarcel, Guy (1999): La Tapisserie Flamande du XVe au XVIIIe siécle, París.

Delmarcel, Guy / Volckaert, An (1995): Tapisseries flamandes. Cinq siécles de tradition. Collection Manufacture Royale De Wit et Bernard Blondeel, Malines.

Delmarcel, Guy / Reyniés, Nicole de / Hefford, Wendy (2010a): La Collection Toms. Tapisseries de XVIe au XIXe siécle, Lausana.

Delmarcel, Guy / García Calvo, Margarita / Brosens, Koenraad (2010b): "Spanish family pride in Flemish wool and silk: The Moncada family and its Baroque tapestry collection". En: Tapestry in the Baroque. New Aspects of Production and Patronage, New York, The Metropolitan Museum of Art-New Haven and London, Yale University Press, pp. 284-315.

De Meûter, Ingrid (2003): "Le peintre anversois Pieter Spierinckx (1635-1711), créateur de cartons de tapisseries". En: Flemish Tapestry in European and American Collections. Studies in Honour of Guy Delmarcel, Turnhout, pp. 133-152.

De Meûter, Ingrid / Vanwelden, Martine (1999): Tapisseries d'Audenarde du XVIe au XVIIIe siécle, Tielt.

Forti Grazzini; Nello (1988): Gli arazzi dei Farnese e dei Borbone, Milano.

García Calvo, Margarita (2011): "Correspondencia entre Fernando de Aragón (1644-1713), 8 duque de Montalto, y su agente en Bruselas sobre la realización de la tapicería de la "Historia de la casa de los Moncada". En: Archivo Español de Arte, LXXXIV, 335, pp. 283-294.

García Calvo, Margarita (2014): "Nuevas noticias sobre dos tapicerías tejidas en la manufactura de Gerard Peemans: Historia de Tito y Vespasiano y Los Meses". En: Archivo Español de Arte, LXXXVII, 345, pp. 75-87.

Hartkamp-Jonxis, Ebeltje / Smit, Hillie (2004): European Tapestries in the Rijksmuseum, Ámsterdam.

Hennel-Bernasikowa, Maria (2000): Gobeliny XV-XIX wieku w zamku krolewskim na Wawelu, Cracovia.

Herrero Carretero, Concha (2008): Vocabulario histórico de la tapicería, Madrid.

Knothe, Florian (2008): "Los cuatro elementos". En: Campbell, Thomas P. (responsable científico): Hilos de esplendor. Tapices del Barroco, cat. exp., Madrid, Patrimonio Nacional, pp. 356-364.

László, Emöke (1981): Tapisseries flamandes et françaises en Hongrie, Budapest.

Ovidio (1997): Metamorfosis, Madrid.

Piwocka, Magdalena (2011): “Tryumf Bachusa i Ariadny”. Sapiehowie. Kolekcjonerzy i mecenasi, Krakow, Zamek Krolewski na Wawelu, pp. 250-251.

Ramírez Ruiz, Victoria (2013): Las tapicerías en las colecciones de la nobleza española del siglo XVII, Madrid, Universidad Complutense.

Reyniés, Nicole de (1997): Charles Poerson, 1609-1667, París.

Schmitz-von Ledebur, Katja (2008a): "Los Meses. Las Estaciones, Los Elementos, El Día y La Noche", en Campbell, Thomas P. (responsable científico). Hilos de esplendor. Tapices del Barroco, cat. exp., Madrid, Patrimonio Nacional, pp. 246-252.

Schmitz-Von Ledebur, Katja, (2008b): "Tapisserien mit mythologischen Darstellungen”, en Vom Mythos der Antike. Catálogo a cargo de Wilfried Seipel. Milán/Viena, 2008, pp. 62-65, nº s. 17-18.

Schmitz-von Ledebur, Katja (2008-2009): "Representaciones mitológicas: Mercurio entrega a Baco a las ninfas para que lo eduquen. Venus y Adonis". En: Jiménez, C. / Calvo Serraller, F.: Todas las historias del arte. Kunsthistorisches Museum de Viena, cat. exp., Bilbao, Museo Guggenheim, pp. 220-223.

Schneider, A. Von (1924): Bemerkungen zu einigen niederlädischen Wirkteppichen des Bayerischen Nationalmuseum, Münchner Jahrbuch der Bildenden Kunst, N.F.I, pp. 70-72, fig. 13.

Thuillier, Jacques (1975): La obra pictórica completa de Poussin, Barcelona.

Van Tichelen, Isabelle / Delmarcel, Guy (1989): Cinq siécles de tapisseries flamandes, Mechelen.

Vittet, Jean / Brejon de Lavergnée, Arnauld (2010): La collection de tapisseries de Louis XIV, Dijon.

Vlieghe, Hans (1959-60): "David Teniers II en David Teniers III als patroonschilders voor de tapijtweverijen". En: Artes Textiles, 5, pp. 78-102.

Wasilkowska, Aleksandra (1971): Gobeliny. Katalog zbiorow, Poznan, Muzeum Narodowe w Poznaniu.

\section{AGRADECIMIENTO:}

Mi agradecimiento a Magdalena Piwocka, al Prof. Guy Delmarcel, a la Dra. Schmitz-Von Ledebur, a Dña ${ }^{\mathrm{a}}$. Patricia Garrido, a Dña. Montse Gañán, a D. Ignacio Bermejo y a los responsables del Archivo de Medina Sidonia.

Fecha de recepción: 13-XI-2014

Fecha de aceptación: 09-II-2015 